6

\title{
Contextual design of management support systems
}

\author{
S. A. Carlsson
}

Department of Informatics

School of Economics and Management

Lund University

Ole Römers väg 6, SE-223 63 Lund, Sweden

Tel.: +4646222 8026, Fax: +46462224528

E-mail: sven.carlsson@ics.lu.se

D. E. Leidner

INSEAD

Technology Management Department

Boulevard de Constance, 77305 Fontainebleau Cedex, France

Tel.: +33160724477, Fax: +33160745500

E-mail:dorothy.leidner@insead.fr

\begin{abstract}
A model for contextual design of Management Support Systems (MSS) is presented. The model is based on Layder's philosophy and four levels framework of social science. Self, situated activity, setting, and context comprise the framework. The model for contextual MSS design can be used to structure, describe, and analyze the context where a MSS is used or is to be used. It can also be used to increase designers as well as other stakeholders understanding of the context where MSS design takes place.
\end{abstract}

\section{Keywords}

Management support systems, design, context, contextual

\section{INTRODUCTION}

There has been emphasis in the literature on information systems (IS) design and IS research on addressing contextual issues (McBride, 1977; Nandhakumar, 1996; Walsham, 1993). Assumptions underpinning this is that a context affects the design and development process of an information system. The context also sets 
the stage for an IS in that it affects what is possible to design and implement. The context also affects IS use and has an impact on effects of IS use.

This paper focuses on contextual design of Management Support Systems. ${ }^{1}$ We, in part, build on previous writings on design of Decision Support Systems (DSS) and Executive Information Systems (EIS) but extend what design and contextual design mean. The presented model for contextual design of MSS builds on Derek Layder's $(1990,1993)$ philosophy and model of social science.

We also build on a view of man based on George Kelly's (1955) 'personal construct theory.' 'It is beyond the scope of this paper to provide more than a brief insight into Kelly's fascinating theory. ${ }^{2}$ Fortunately, the basic ideas of personal construct theory are comprehensible on an 'intuitive' basis. These basic ideas are presented. In a formal way, Kelly presented his theory as a fundamental postulate and elaborations of it by means of eleven corollaries. The fundamental postulate states: 'A person's processes are psychologically channelized by the ways in which he anticipates events' (Kelly, 1955, p. 46). Kelly viewed man as a personal scientist, as man-the-scientist '...classifying, categorising and theorising about his world, anticipating on the basis of his theories and acting on the basis of his anticipation' (Shaw, 1980, p. 7). In Kelly's own words:

'Now what would happen if we were to reopen the question of human motivation and use our long-range view of man to infer just what it is that sets the course of his endeavor? ...Might not the individual man, each in his own personal way, assume more of the stature of a scientist, ever seeking to predict and control the course of events with which he is involved? Would he not have his theories, test his hypotheses, and weigh his experimental evidence? And, if so, might not the differences between the personal viewpoints of different men correspond to the differences between the theoretical points of view of different scientists?' (p. 5).

Kelly's view of man is anti-positivistic in its epistemology. He is concemed with how a person models his experiences and, based on this model, how he anticipates the future. Such anticipation might be active, as in action, or passive, as in prediction. Kelly also points out that a person's models are both cognitively and socially constructed. ${ }^{3}$ Based on Kelly's view of man, we perceive designers as well as other stakeholders involved in MSS design and MSS use as being 'scientists.' They will develop their own theories (mental models) about, for example, MSS use and affects of MSS use.

\footnotetext{
${ }^{1}$ By the term Management Support Systems we mean computer-based systems that purport to support managers in Type II work (Sprague, 1986; Sprague \& Watson, 1996). MSS includes Decision Support Systems and Executive Support Systems in a wide sense.

2 For more extensive introductions to Kelly's theory, the reader is recommended to read Bannister and Fransella (1986), Kelly (1970), or Winter (1992, Chapter 1 \& 2).

3 A similar view of man is expressed by Galliers and Swan (1997): 'A subjective, pluralistic stance views perceptions of the 'real' world as cognitively and socially constructed. Individuals engage in sensemaking when dealing with complex problems and use unique cognitive schema (mentally represented concepts and relationships among concepts) to construct and simplify problems.' (p. 182)
} 
The remainder of the paper is organized as follows. The next section describes some current DSS and ESS approaches and points out that they seem to lack certain critical elements. The basis of Layder's philosophy and framework is presented in Section 3. A section that presents Layder's four levels framework and how it can be used in contextual MSS design follows it. In the final section implications and conclusions are presented and discussed.

\section{CURRENT MSS DESIGN APPROACHES}

Over the years writers in the DSS field have addressed design issues. Huber (1980) contributed by distinguishing between 'dss' and 'DSS,' where only the latter, he suggests, are computer-based systems. He pointed out that decision support is a vital part of management in all organizations. Huber also suggested that a key requirement for effective decision support is that the DSS fit the manager's dss. Stabell (1986, 1987), building on Huber's distinction and Simon's (1981) idea about the sciences of the artificial, defined DSS as decision support by design. Stabell identified and described four DSS design school: decision analysis, decision calculus, decision research, and implementation process. Stabell points out that in order to design a DSS, the context has to be understood. Silver (1991) proposed ways to describe DSS and how to differentiate them one from another. Silver suggests that good prescription for DSS design depends on good and systematic descriptions and these, in turn, depend on a common 'language' for describing and differentiating DSS. He proposed system design dimensions like restrictiveness, guidance, and customizability. More recently, Dutta et al. (1997) proposed a MSS design approach based on Silver's system design dimensions together with the objects of decision support (outcome, process, or learning) and the modes of decision support (automate, informate, or stimulate).

In the EIS field, Rockart (1979) proposed a critical success factors (CSF) approach to identify executives information needs. Critical success factors are '...the few key areas of activity in which favorable results are absolutely necessary for a particular manager to reach his goals' (Bullen and Rockart, 1981). The CSF approach was extended to include critical decisions and critical assumptions (Henderson et al, 1987). Critical decisions are the decisions or decision processes that directly affect the success or failure of the CSF. Critical assumptions are the assumptions that lead an individual to believe that the CSFs are valid. Volonino and Watson (1990-91) proposed a strategic business objectives (SBO) approach. The SBO approach links executive information needs to an organization's goals and objectives. It consists of six steps. The early ones being: identify an organization's strategic business objectives, identify business processes that are critical to the business objectives, prioritize strategic business objectives and critical business objectives, and define information needed to support the critical business processes.

The CSF and the SBO approaches build on management theories and especially strategic management theories. The approaches focus on information needs and 
especially monitoring information. Walls et al (1992) proposed a theory for designing vigilant EIS. A vigilant EIS is an information system that helps '...an executive remain alertly watchful for weak signals and discontinuities in the organizational environment relevant to emerging strategic threats and opportunities' (p. 36). The vigilant EIS approach also focuses on information needs since it provides a design to support executives in their tracking of issues. The authors also propose an information requirement determination method called the 'Critical Attention Tracker' approach.

All the above design approaches and methods are useful but they seem to lack certain critical elements. Examining the design approaches, it should be no surprise that we find most of them to be based on a positivistic paradigm. However, researchers have articulated the need for design approaches building on nonpositivistic paradigms (Hirschheim and Klein, 1989). livari (1991) analyzed the fundamental assumptions of seven major schools of thought in IS design: software engineering, database management, management information systems, decision support systems, implementation research, sociotechnical approach, and infological approach. He writes that the schools '...indicate a uniform adherence to the positivistic epistemology of regularities, causal laws and explanation.' (ibid., p. 262). livari points out that DSS has some ideas, which may be interpreted as antipositivistic, but more research is needed on non-positivistic design approaches and models. Galliers and Swan (1997) point out that almost all IS design approaches adopt a unitary, objective perspective. This is due to the approaches' positivistic stance. The MSS design model we present in this paper is non-positivistic.

Recent research on use of EIS suggests the need for a wider perspective on what is meant by context. EIS can be considered as systems that give its users access to needed information. From this perspective the CSF and SBO approaches seem to be practicable since they have an information providing perspective. But, more recent research suggests that EIS, and in general MSS, are systems that are used to support different organizational processes and managerial activities. Vandenbosch and Higgins (1995) found that EIS are used in both single-loop and double-loop learning and Carlsson et al (1996) found that EIS are used to, in part, drive organizational changes and motivate employees. The word context denotes that the whole situation, background, or environment relevant to something has to be considered.

The above mentioned studies suggest that MSS designers and MSS design approaches have to have a broader perspective on what is meant by context. We present a model that has a broader perspective on what is meant by context in MSS design.

Finally, the points made above and the type of users supported by MSS lead to certain issues regarding the relationships between designers, users, and other stakeholders. In general, the writings on IS development recommend that users should be involved in the design process. A problem often encountered in the development of MSS is that the users have very limited time for participating in 
the design process (Watson et al, 1997; Nandhakumar and Jones, 1997). This puts an extra burden on MSS designers. They must be very aware about the context in two respect: 1) the context a MSS is designed to be used in, and 2) the context of the MSS design process.

To overcome some of the abovementioned problems and weaknesses we propose a contextual MSS design model. The model is based on Layder's framework of social organization. Layder's framework says that, in social studies, like MSS design, four levels have to be addressed: self, situated activity, setting, and context. Self refers to an individual's relation to her or his social environment. Situated activity focuses on the dynamics of social interaction. Setting focuses on the intermediate forms of social organization and the wider macro social forms that provide the more remote environment of social activity is referred to as the context. It is important to note that all four levels refer to context. Still, we will use Layder's concepts since they help us distinguish between different elements of what constitutes a context.

The model we present can be used in the MSS design process for describing the context a MSS is designed, for and for assessing, MSS use. The model can also be used to increase designers and other stakeholders understanding of the context within which MSS design takes place.

\section{GOING BEYOND POSITIVIST APPROACHES}

In recent years, several researchers have pointed out the need for non-positivistic studies, models, and approaches in the IS field (Hirschheim and Klein, 1989; Orlikowski and Baroudi, 1991; livari, 1991; Walsham, 1993, 1995). Lee (1991) suggests that IS and organization researchers should integrate positivist and interpretive approaches. Although Lee's suggestion has some similarities with what we propose, in this paper, his proposed model is not based on a specific philosophy of social sciences. The model we propose is based on Layder's philosophy and framework of social science (Layder, 1990, 1993). Layder's work belongs to what is called the realist project. The realist project was developed as an alternative to traditional positivistic models of social science (Layder, 1990). ${ }^{4}$ As Layder (1990) writes,

'Put very simple, a central feature of realism is its attempt to preserve a 'scientific' attitude towards social analysis at the same time as recognizing the importance of actors' meanings and in some way incorporating them in research. As such, a key aspect of the realist project is a concern with causality and the identification of causal mechanisms in social phenomena in a manner quite unlike the traditional positivist search for causal generalizations.' (p. 16).

4 Different aspects of the realist project can be found in Layder (1990, 1993, 1994), Harré and Secord (1972), Keat and Urry (1981), and Bhaskar $(1989,1994)$. 
From an epistemological stance, concerning the nature of knowledge claim, the realist approach is non-positivistic which means that values and facts are intertwined and hard to disentangle.

Several IS writers have pointed out to grounded theory (GT) as an alternative to positivistic approaches. Although positive to Glaser and Strauss's (1967) grounded theory (GT), Layder (1993) points out major weaknesses in grounded theory such as that the GT approach

'... must break away from its primary focus on micro phenomena. The very fixity of this concentration is a factor which prevents grounded theory from attending to historical matters of macro structure as a means of enriching contemporary or, as I [Layder] shall call them, present-centred forms of research on micro phenomena. It should be possible to augment the processual and dynamic analyses of interactional phenomena by a parallel focus on the historically antecedent forms that provide their institutional backdrop.' (p. 68).

Transferred to IS research and design Layder's comments mean that researchers and designers that base their work on grounded theory must identify the influence of macro structural features on behavior and interactions in what they are able to 'observe' directly. In other words, macro phenomena have no validity to 'GT designer and researchers' unless they could be derived directly from field data. But, research suggests that macro phenomena, like national culture, influence IS designers (Hunter and Beck, 1996) and how IS are used and are assessed (Tan et al, 1995; Leidner et al, 1997).

Other weaknesses of the grounded theory approach pointed out by Layder include: how power can be handle in it. Grounded theory focuses on situated and interpersonal aspects. This means that GT misses the importance of power 'behind the scenes' of activity. Group Support Systems (GSS) researchers stress the importance of anonymous communication as a way to support equal participation and even out power differences. In most cases, this is discussed without noting the setting, that is, without discussing the power structure in the setting that, ultimately, has an impact on a decision group's process activities and decision implementation possibilities. To put it differently, by neglecting the setting one misses the interplay between structural and interactional dimensions of power. The importance of structural dimensions in understanding the relationships between IS design, IS use and power has been pointed out by Markus and Bjøm-Andersen (1987) and Davenport and Prusak (1997).

Layder also borrows some ideas from middle-range theory (Merton, 1967). This to, a minor extent, than from GT. He points out the strengths as well as the weaknesses of middle-range theory. A strength is that it focus on macro phenomena while a weakness is its rigid notion of social science (positivistic) which is quite different from what Layder presents.

Layder developed his framework primarily for being used in theory generation and development and less so for theory testing. This framework can be used for 
generating both substantive and formal theories. The former is theory 'developed for a substantive area such as patient care, race relations, professional education, geriatric lifestyles, delinquency or financial organizations' and formal theory is theory "developed for a formal or conceptual area of sociological inquiry such as status passage, stigma, deviant behaviour, socialization, status congruency, authority and power, reward systems, organizations or organizational careers.' (Glaser and Strauss, 1971, p. 177-178). Layder suggests that his framework can also be used in studies which have information gathering or descriptive aims.

Having presented the basis of Layder's model, next section presents a nonpositivistic model of MSS design based on Layder's model.

\section{A NON-POSITIVISTIC MODEL OF MSS DESIGN}

Layder suggests a stratified or layered framework of social organization. The framework includes macro phenomena, like structural and institutional phenomena, as well as micro phenomena, like behavior and interaction. The outline of our contextual MSS design model is presented in Figure 1 which describes levels (elements) of potential areas of interest in contextual MSS design. As the figure suggests, Layder's framework reflects a greater appreciation of the multifaceted nature of the empirical world than do the middle-range theory and the grounded theory. These tend to exclusively focus on one or two of the elements.

We will briefly present the different elements and, then, discuss some of the implications for contextual MSS design. We will, for convenience, start with the self and work towards the macro elements. The first level is self, which refers '... primarily to the individual's relation to her or his social environment and is characterized by the intersection of biographical experience and social involvements.' (Layder, 1993, p. 9). Self focuses on how an individual is affected by and responds to social situations. In encountering social situations, individuals use strategies, based on their 'theories' (mental models), to handle the situations. These strategies and how they are used can be addressed. How individuals, emotionally and intellectually, perceive, describe, and understand specific situations are also important areas to address. In general, the self and situated activity have as their main concern '...the way individuals respond to particular features of their social environment and the typical situations associated with this environment.' (Layder, 1993, p. 79).

In situated activity, the focus is on the dynamics of social interaction. The area of self focuses on how individuals are affected and respond to certain social processes whereas situated activity focus on the nature of the social involvements and interactions. This means that the interaction and the process have features that are the result of how the participating individuals' behaviors intermesh and coalesce. Situated activities vary considerably along a number of dimensions (for example, along the dimensions 'time' and 'place'). 


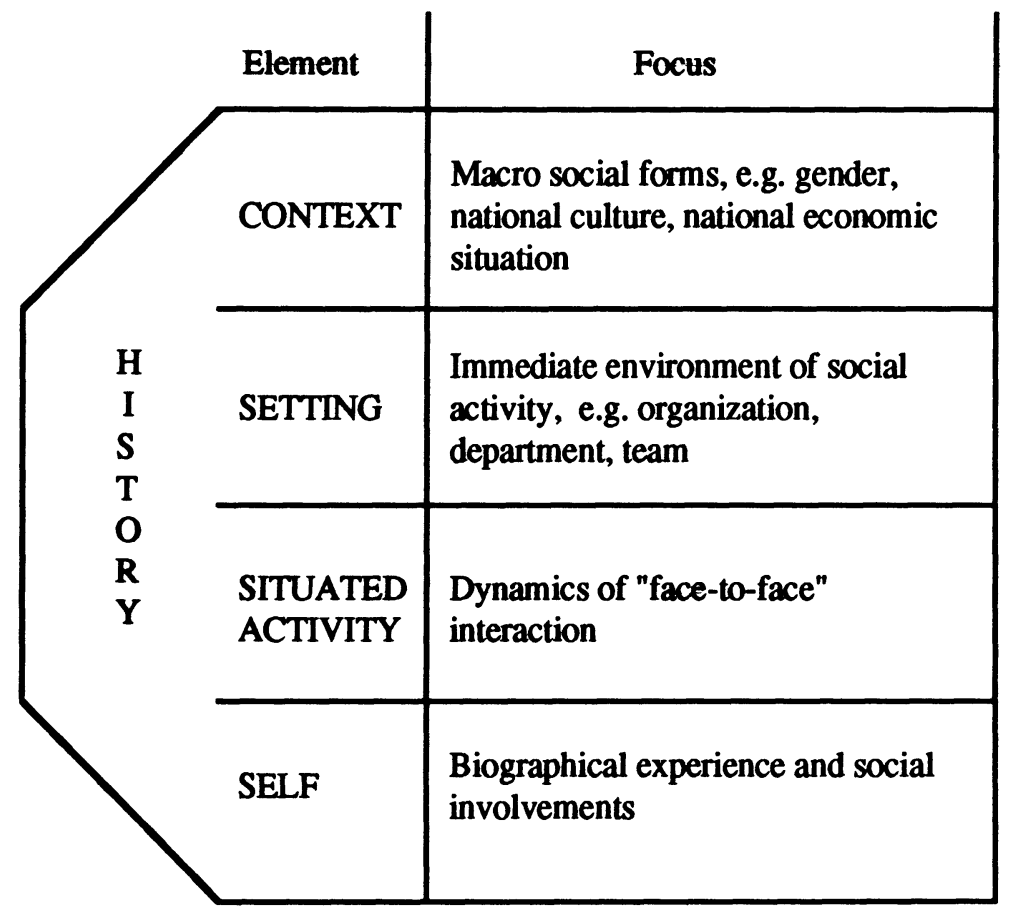

Figure 1 Contextual MSS design model (adapted from Layder, 1993, p. 8).

Self and situated activity can be treated as separable elements in contextual design but they are, in practice, hard to separate. It is hard to separate selves from the social situation in which they are embedded, but, by separating them, a designer or researcher can focus on: 1) how individuals respond to, and are affected by, their social interactions and situations, and 2) the nature of the social interactions themselves. For example, how users react to a specific type of computer-mediated communication and the nature of the computer-mediated communication. The topics addressed at the self and situated activity levels can be subjective as well as objective 'components and characteristics'.

The focus in setting is on the intermediate forms of social organization. A setting provides the immediate arena for social activities. A setting can be things like the culture of the organization, artifacts (like Management Support Systems) that are used in situated activities, power and authority structures. It should be stressed that setting is not just a particular pattem of activity. Seeing setting and context as a particular pattern of activity is a characteristic feature of GT and other approaches that draw on an interactionist perspective. Layder sees setting and context as '...rather different but complementary aspects of social life, and that, in 
principle, full and equal weighting should be given to each in field research.' (Layder, 1993, p. 89).

The wider macro social forms that provide the more remote environment of social activity are referred to as the context. Although there is not a clear border between settings and context and some social forms straddle the two elements, it can be fruitful to distinguish them. In general, context refers to large-scale and society-wide features. Macro elements that have been used in IS studies include gender, national culture, and national economic situation.

Viewing MSS design as layers of social organization that are interdependent has two major advantages. It enables a designer, and other stakeholders, to be sensitive to the different elements with full appreciation of their distinctive features. In reviewing some MSS design approaches and models, we have found that, in general, they do not distinguish between the different layers. They handle features of the different layers in the same way and they do not express their underlying assumptions about the interdependence between the layers other than in fairly simple causal relationships. The MSS design model presented here also stresses that the layers operate on different 'time scales.' For example, computer-based interaction, using a MSS, is a social activity (a situated activity) that, within a certain time span, is a continuous process. In assessing the MSS, a designer has to understand the way such a process unfolds over time and is related to the other elements and their changes over time. This means that the designer has to view the operation of the elements not only vertically but also horizontally.

\subsection{A model for contextual management support systems design}

Having briefly presented Layder's framework and the contextual MSS design model proposed we now turn to consider how it can be used. The design model can be used in, at least, four different ways.

- It can be used in a MSS design process to support stakeholders in their development of 'theories' of the context a MSS is designed for.

- For understanding a MSS design process by supporting stakeholders in identifying and describing the different elements and dimensions that constrain the process.

- For organizing and assessing empirical MSS research, especially, effect and impact studies. The model can be used in setting up a field study, which has as its primary goal theory generation. It can be used for generating research questions, as help in organizing the research, and for doing selective focusing. The latter concerns the choice of an uneven focus, which means that a researcher decides to emphasise particular elements for practical or theoretical reasons. Once the research gets going, the model can be used in the development of emergent theory. The model can also be used as a sounding board for theoretical ideas in cases where a research project has come to a dead end. 
- As a way of organizing, understanding, and assessing MSS design approaches and models. Since this is not the main purpose of the resource model it should be stressed that the model is one and only one way to organize and understand previous work.

In this paper we will mainly address the first two concerns.

\section{Contextual Management Support Systems design}

When a designer focuses on self and situated activity, four major issues can be addressed. First, which stakeholders are or can be affected by a MSS. Secondly, how the different stakeholders are affected by the MSS. Thirdly, how MSS users and other individuals affected respond to particular type of system characteristics and features Fourthly, what meanings, perceptions, and assessments are associated with certain ways to use the MSS. How are these meanings, perceptions, and assessments influencing the situated activities? Are there changes over time in meanings, perceptions, and assessments? If there are changes, what causes these changes?

The four issues are, to some extent, normally addressed in MSS design, but we have to point out that, in order to properly address the issues, a designer has to at least 'keep control' of the other elements in the design map. For example, situated activity, setting, and context might heavily influence how an individual perceives certain features of a MSS.

As discussed above, the different elements operate in two different dimensions (vertically and horizontally). Vertically, as a series of layers, and, horizontally, as layers stretched out over time. In reality, the dimensions operate simultaneously. This has consequences for MSS design. We can use three examples as illustrations. In the first example, imagine a designer who is developing a MSS to be used for communicating certain types of information. The designer can use a selective focus by focusing on the situated activity and try to understand how the dynamics of interaction change due to the MSS. In this situation, the designer's focus is on situated activity but he has to understand the role the setting can play and also have a focus on the self, (for example, how the users are affected by the change). The time scale in this case can be from a couple of weeks to years. In the second example, imagine a designer who is interested in testing how a new analysis capability in a MSS influences an individual decision making process. The focus could be on the self and the situated activity. The time frame can be a couple hours but the designer can also study the changes over a more extended time period. The latter can be motivated by research suggesting that there is a learning effect. In the third example, igamine a designer and other stakeholders who are interested in if and how a MSS can influence an organization (the setting) (for example, if it can be used to support a change in the power structure). In this case, the designer and the stakeholders are interested in how the two elements, situated activity and setting, interweave. The three examples illustrate that a designer has a choice in terms of 
what elements (layers) to focus on and for how long. This choice should, in an ideal situation, be 'theoretically' based.

A designer focusing on the setting can address issues like how different aspects of the setting can influence situated activity or in what specific setting it is likely that a certain type of outcome will be the effect of using a specific MSS. In general, it is essential to know the social relationships that exist in particular settings to be able to understand the situated activities. Normally, setting receives little attention in MSS design. A designer might consider the formal aspects of the setting, but issues of commitment and attachments are seldom addressed. A MSS designer interested in situated activities should assess and understand what commitments and attachments individuals have in the particular setting; how commitments can influence the situated activities as well as how the setting (in terms of commitment) can be influenced by changes in situated activities (for example, by the use of a MSS). Other important characteristics of setting are authority and power. These are working 'behind the scene' of the situated activity and might have major impacts on situated activities.

It seems that most writings on MSS design approaches neglect the context. As discussed above, it seems that national culture can play a role and it is an aspect that more and more organizations have to consider in an increasing global economy. Other aspects like norms, values, codes of behavior, and gender can also be addressed. Research suggests that some of the old MSS truths do not hold in non-Anglo-American cultures (Leidner et al, 1997; Carlsson et al, 1996; Kosaka and Fitzgerald, 1977).

Settings and contexts differ in their continuity and durability, that is, they operate on different time scales. With respect to MSS design it is likely that the contextual aspects will influence the immediate settings and the situated activities. It is less likely that changes in the situated activities can, due to MSS use, lead to changes in the contexts (at least not in the short run). The settings can influence the situated activities and changes in the situated activities can, due to MSS use, lead to changes in the settings.

\section{Context of Management Support Systems design}

A MSS design process 'takes place' in a context. It is a contextual process that is 'connected with, or depending on the context' (Webster's New Twentieth Century Dictionary). The design process is a situated activity and different stakeholders participate in the process. An important goal in the design process is system requirement specification. As stressed, this stage, as well as other stages, is a socially mediated process (Galliers and Swan, 1997). In general, the literature on MSS design provides strategies and tactics to be used by designers to increase the likelihood of developing a MSS that will be used and that the users will find useful. These tactics and strategies concem micro phenomena, that is, behavioral and interactional phenomena. Very few address macro phenomena like power 
structure and national culture. These phenomena can constrain a designer in terms of what is possible with regards to action and interaction.

The proposed model can be used by designers to describe and understand the MSS design process context. Hence, the use of the model can increase a designer's understanding. It should be possible for a designer to act more consciously and deliberately and, hence, to increase the likelihood of getting a 'good' process.

Layder clearly points out that the elements he describes are not fixed (he has a non-deterministic view). Hence, the elements are not producing certain outcomes but they can constrain a designer. The elements are not only constraining a designer, they are also opportunities for action. Certain dimensions of the elements are limiting certain types of action but, at the same time, they are enabling others. As said by Giddens: 'All types of constraint ... are also types of opportunity, media for the enablement of action. ' (Giddens, 1984, p. 117). In a specific design process, all elements are not equally important. The model can be used by a designer to be guided as to which elements and dimensions to address and which to neglect.

\section{IMPLICATIONS AND CONCLUSIONS}

The original contribution of this paper is the development of a new conceptualization of contextual MSS design. The presented model represents a broader perspective on contextual MSS design.

The presented model is non-positivistic drawing upon Layer's model and borrows from two main approaches, grounded theory and middle-range theory, and, hereby, overcomes the shortcomings of the two approaches. This is done by focusing on: 1) the layered nature of social processes, 2) the unfolding nature of social activity over space and time, and 3 ) the integrated nature of micro and macro features.

In contextual MSS design the model can be used to: 1) support designers and other stakeholders in describing, understanding, and analyzing the context a MSS is designed for, and 2) describe and understand a MSS design process by supporting designers and other stakeholders in identifying and describing the different dimensions that 'constrain' the design process.

Based on Layder's framework and our MSS design model, we suggest some practical MSS design recommendations. Writers have suggested that IS/MSS designer should try to increase the depth of qualitative analyses. Layder's framework suggests several ways to address this. In order to achieve a dense empirical coverage, designers should use a multistrategy approach. This means that a designer should use as many analytical and methodological cuts into the data as possible. (Of course, considering constraints in terms of, for example, time and costs). We are not advocating an 'anything goes' or eclectic approach. On the contrary, we suggest disciplined flexibility, which means that the theoretical assumptions which underlie the MSS design model should guide the selection of 
methods. Also, the overarching vision of the relations between micro and macro elements requires MSS designers to use both quantitative and qualitative data. Quantitative and qualitative data are viewed as complementary to each other.

Another recommendation is to use 'triangulation.' In general, triangulation means the use of a combination of methods and techniques to confirm or disconfirm findings arrived at by using a particular method or technique. (It should be noted that there exist other definitions of triangulation and related concepts.) Triangulation in contextual MSS design is used for reliability, validity, and generality tests of findings. These findings can be, for example, assessment of MSS use or anticipated use. Layder's framework has, by its multistrategy approach, built triangulation into it.

The issue of longitudinallity (the horizontal perspective) can, in many cases, be a problem. Layder's framework clearly points out the importance of history and the understanding of how things evolve over time. In most cases, designers can not focus on all elements and dimensions. Designers must have a selective focus. Hence, addressing specific design questions, longitudinally can mean quite different things. For example, if a purpose of a MSS is to support an organization in changing its organizational culture, longitudinal could mean a couple of months or years. On the other hand in testing a new MSS feature, longitudinally can mean a couple of hours or days. Our recommendation is that designers should be aware and try to understand what longitudinally means in their specific design situation.

It is often recommended that MSS designers should use prototypes in the design process (Volonino and Watson, 1990-91; Watson et al, 1997). This is like conducting lab studies during the design process. Although we are positive to the use of prototypes, we like to recommend MSS designers to use the MSS design map to understand and articulate what they are gaining as well as what they are missing when creating less than realistic social situations and processes. The design map can help designers understand what issues can be studied using prototypes and what issues do not lend themselves to be studied and assessed using prototypes.

The last recommendation is that designers should see themselves as designers of artificial organizational processes and not just designers of the artifacts (i.e., the MSS). In using the realist approach in MSS design a designer has to adapt it to the specific situation at hand. A designer is, in part, designing artifacts (MSS) as well as artificial organizational processes. In implementing a MSS in an organization, a designer is, more or less, deliberately designing organizational processes - that is, the organizational processes do not evolve naturally. This means that it is important to raise the issue of why a MSS and organizational processes should be designed and implemented in the first place. What are the goals and intentions of the designers? Are there other stakeholders, and what are their intentions, etc.? Designing computer-based systems is quite a unique situation in the social science in that designers are 'studying' processes in order to understand the impact of the 
use of artifacts and artificial systems to be able to design such systems better in the future.

Layder's model has also research implications. Although we have only given a glimpse of the model in this paper, we think that it can also provide a useful reference point for MSS researchers wishing to work in the non-positivistic tradition. The approach encourages careful work on the conceptualization and execution of empirical MSS studies. There exist several research avenues to be taken. One is to develop more specific contextual MSS design theories. As should be clear, research based on Layder's model does not develop theories that show how two or more variables relate to each other. Instead, it will be theories that are regarded as 'integrated clusterings' or 'networks' of concepts, propositions and 'world-views' (Pawson, 1989; Layder, 1990).

In contrast to GT, the realist approach encourages the use of theory in research. Based on Layder's suggestions, we can advice researchers doing empirical theory generating research on contextual MSS design to use at least sensitizing or background concepts. A researcher can also aim towards the construction of typologies as an aid to generate theory. In terms of typologies, Glaser and Strauss are positive to the development of what Layder calls action typologies (related to self and situated activity) but Layder suggests that the development of structural typologies (related to setting and context) should also be done. The development of typologies can also serve as benchmarks for theoretical sampling and comparative analysis. There is also the possibility to use theories and constructs about constraints on human action and interaction. Based on Giddens' (1984) work, Nandhakumar and Jones (1997) and Kosaka and Fitzgerald (1997) have presented such ideas that seem worthwhile to explore.

\section{ACKNOWLEDGEMENTS}

The authors appreciate the helpful comments and encouragement by Dina Berkeley and three anonymous reviewers.

\section{REFERENCES}

Bannister, D. and Fransella, F. (1986) Inquiring man: the psychology of personal constructs. Third ed., Penguin, Harmondsworth, England.

Bhaskar, R. (1989) Reclaiming reality. Verso, London.

Bhaskar, R. (1994) Plato etc. Verso, London.

Bullen, C.B. and Rockart, J.F. (1981) A primer on critical success factors. CISR Working Paper 69. Center for Information Systems Research, Sloan School of Management, Massachusetts Institute of Technology, Cambridge, MA. 
Carlsson, S.A., Leidner, D.E. and Elam, J.J. (1996) Organizational and individual effectiveness: perspectives on the impact of ESS in multinational organizations, in Implementing systems for supporting management decisions (eds. P. Humphreys, L. Bannon, A. McCosh, P. Migliarese and J.-C. Pomerol), Chapman and Hall, London, 91-107.

Davenport, T.H. and Prusak, L. (1997) Information ecology. Oxford University Press, Oxford, UK.

Dutta, S., Wierenga, B. and Dalebout, A. (1997) Designing management support systems using an integrative perspective. Communications of the $A C M, 40$, 6, 70-79.

Galliers, R.D. and Swan, J.A. (1997) Against structured approaches: information requirements analysis as a socially mediated process. Proceedings of the Thirtieth Hawaii International Conference on System Sciences, Vol. III, IEEE Computer Society Press, Los Alamitos, CA, 179-187.

Giddens, A. (1984) The constitution of society. Polity Press, Cambridge, UK.

Glaser, B.G. and Strauss, A.L. (1967) The discovery of grounded theory. Aldine, Chicago, IL.

Glaser, B.G. and Strauss, A.L. (1971) Status passage. Routledge, London.

Harre, R. and Secord, P. (1972) The explanation of social behavior. Blackwell, Oxford, UK.

Henderson, J.C., Rockart, J.F. and Sifonis, J.G. (1987) Integrating management support systems into strategic information systems planning. Journal of Management Information Systems, 4, 1, 5-24.

Hirschheim, R. and Klein H.K. (1989) Four paradigms of information systems development. Communications of the ACM, 32, 10, 1199- 216.

Huber, G. (1980) Organizational science contributions to the design of decision support systems, in Decision support systems: issues and challenges (eds. G. Fick and R.H. Sprague), Pergamon Press, London, 45-55.

Hunter, M.G. and Beck, J.E. (1996) A cross-cultural comparison of 'excellent' systems analysts. Information Systems Journal , 6, 4, 261-281.

livari, J. (1991) A paradigmatic analysis of contemporary schools of IS development. European Journal of Information Systems, 1, 4, 249-272.

Jones, M. and Nandhakumar, J. (1993) Structured development? a structurational analysis of the development of an executive information systems, in Human, Organizational, and Social Dimensions of Information Systems Development (eds. D. Avison, J.E. Kendall and J.I. DeGross), North-Holland, Amsterdam, 475-496.

Keat, R. and Urry, J. (1981) Social theory as science. RKP, London.

Kelly, G.A. (1955) The psychology of personal constructs. W.W. Norton, New York, NY.

Kelly, G.A. (1970) A brief introduction to personal construct theory, in Perspectives in personal construct theory (ed. D. Bannister), Academic Press, London: 1-29. 
Kosaka, T. and Fitzgerald, G. (1997) Structurational analysis of comparative study of EIS between the United Kingdom and Japan. Proceedings of the Eighteenth International Conference on Information Systems, Atlanta, GA, December 1517, 496-497.

Layder, D. (1990) The realist images in social science. Macmillan, London.

Layder, D. (1993) New strategies in social research. Polity Press, Cambridge, UK.

Layder, D. (1994) Understanding social theory. Sage, London.

Lee, A.S. (1991) Integrating positivist and interpretive approaches to organizational research. Organization Science, 2, 4, 342-365.

Leidner, D.E., Carlsson S.A., Elam, J.J. and Corrales, M. (1997) A multicultural perspective of the impact of EIS on organizational intelligence, decision making, and structure. Working Paper, INSEAD, Fontainebleau.

Markus, M.L. and Bjom-Andersen, N. (1987) Power over users: its exercise by system professionals. Communications of the ACM, 30, 6, 498-504.

McBride, N. (1997) The rise and fall of an executive information systems: a case study. Information Systems Journal, 7, 4, 277-287.

Merton, R. (1967) On theoretical sociology. Free Press, New York, NY.

Nandhakumar, J. (1996) Design for success?: critical success factors in executive information systems development. European Journal of Information Systems, $5,62-72$.

Nandhakumar, J. and Jones, M. (1997) Designing in the dark: the changing userdeveloper relationship in information systems development, in Proceedings of the Eighteenth International Conference on Information Systems, Atlanta, GA, December 15-17, 75-87.

Orlikowski, W.J. and Baroudi J.J. (1991) Studying information technology in organizations: research approaches and assumptions. Information Systems Research, 2, 1, 1-28.

Pawson, R. (1989) A measure for measures. Routledge, London.

Rockart, J.F. (1979) Chief executives define their own data needs. Harvard Business Review, 57, 2, 81-93.

Shaw, M.L.G. (1980) On becoming a personal scientist. Academic Press, London.

Silver, M.S. (1991) Systems that support managers: description and analysis. Wiley, Chichester, UK.

Simon, H.A. (1981) The sciences of the artificial. Second edition, MIT Press, Cambridge, MA.

Sprague, R.H., Jr. (1986) DSS in context, in Decision support systems: a decade in perspective (eds. E. McLean and H.G. Sol), North-Holland, Amsterdam, 19-24.

Sprague, R.H., Jr. and H.J. Watson (1996) Decision support for management. Prentice Hall, Upper Saddle River, NJ.

Stabell, C.B. (1986) Decision support systems: alternative perspectives and schools, in Decision support systems: a decade in perspective (eds. E. McLean and H.G. Sol), North-Holland, Amsterdam, 173-182. 
Stabell, C.B. (1987) Decision support systems: alternative perspectives and schools. Decision Support Systems, 3, 243-251.

Tan, B.C.Y, Watson, R.T. and Wei, K.-K. (1995) National culture and group support systems: filtering communication to dampen power differentials. European Journal of Information Systems, 4, 82-92.

Vandenbosch, B. and Higgins, C.A. (1995) Executive support systems and learning: a model and empirical test. Journal of Management Information Systems, 12, 2, 99-130.

Volonino, L. and Watson, H.J. (1990-91) The strategic business objectives method for guiding executive information systems development. Journal of Management Information Systems, 7, 3, 27-39.

Walls, J.G., Widmeyer, G.R. and El Sawy, O.A. (1992) Building an information system design theory for vigilant EIS. Information Systems Research, 3, 1, 36-59.

Walsham, G. (1993) Interpreting information systems in organizations. Wiley, Chichester, UK.

Walsham, G. (1995) Interpretive case studies in IS research: nature and method. European Journal of Information Systems, 4, 1, 74-81.

Watson, H.J., Houdeshel, G. and Rainer K., Jr. (1997) Building executive information systems and other decision support applications. John Wiley and Sons, New York.

Weick, K.E. (1979) The social psychology of organizing. Second edition, Random House, New York, NY.

Winter, D.A. (1992) Personal construct psychology in clinical practice: theory research and applications. Routledge, New York, NY.

\section{BIOGRAPHIES}

Sven A. Carlsson, Department of Informatics, Lund University, Sweden, has a Ph.D. in Informatics from the School of Economics and Management at Lund University. His research interests include the use of information technology to support both individual and group decision making, knowledge management, strategic information systems, and organizational transformation through information technology. He has been a visiting scholar at University of Arizona, Tucson, University of Southern California, and National University of Singapore. His articles have appeared in Journal of Management Information Systems, Information and Management, Journal of Decision Systems, Scandinavian Journal of Information Systems, and international conference proceedings.

Dorothy E. Leidner is Associate Professor of Information Systems at INSEAD, Fontainebleau, France. She received her BA, MBA, and Ph.D. from the University of Texas at Austin. Her research interests include executive information systems, 
the impact of IS on executive decision making, virtual organizational forms, international information systems, and the use of IT in education. She has publications in Information Systems Research, Journal of Management Information Systems, Decision Support Systems, MIS Quarterly, Organization Science, and other IS journals and international conference proceedings. 